\title{
HEMOTHORAX - A COMPLICATION OF SUBCLAVIAN VEIN CANNULATION
}

\author{
Jan Harrer, Miroslav Brtko, Pavel Žáček, Ján Knap \\ Department of Cardiac Surgery, University Teaching Hospital, Hradec Králové; \\ (Head: doc. MUDr. J. Dominik, CSc.)
}

Summary: Massive bleeding into pleural cavity after subclavian vein cannulation is a rather rare but very serious complication. Usually laceration of the venous wall is the cause. In patients where conservative treatment, i.e. pleural drainage, maintaining the circulatory volume, treatment of possible coagulopathy, etc. is ineffective, surgery has to be performed. Bleeding can be surgically managed either from posterolateral thoracotomy or direct subclavian vessel revision is possible after partial resection of the clavicle. Brachiocephalic vein bleeding can be approached and managed through median sternotomy. We present a case report of 22-year old man with hemothorax after subclavian vein cannulation. In our patient only complex surgical procedure enabled proper management of bleeding complication.

Key words: Central venous line; Complications; Hemothorax; Subclavian vein

An intensive-care patient could hardly be managed properly without a central venous line. Usually subclavian vein is cannulated for this purpose. Aubaniac first described this technique in 1952 (1).

Pneumothorax is the most frequent serious complication of subclavian vein cannulation. The incidence varies from $1-3,5 \%$ according to the physician's experience and skills $(2,3)$. Much less frequent but more serious complications are hemothorax and hemomediastinum. With minor bleeding these complications may remain clinically silent. Serious bleeding with severe blood loss leads to hypovolemia, the signs of which may appear quite late after the cannulation. The patient's condition can deteriorate into a critical state of hemorrhagic shock. Mainly laceration of subclavian vein can be the cause. Another source of hemothorax can be subclavian artery laceration. The incidence of arterial puncture during subclavian vein cannulation is reported to be approximately $4 \%$ (2).

To confirm the diagnosis of hemothorax, chest X-ray is helpful. We can find pleural effusion, widening of upper mediastinum or dislocation of the trachea. Sometimes we can see a „pleural cap“ shadow in the pleural cupula which is the sign of extrapleural hematoma.

\section{Case report}

22-year old man was referred for urgent dialysis because of suspected methanol intoxication. For that reason a double lumen dialysis cannula $(10,8 \mathrm{~F})$ was inserted into his left subclavian vein. The bicarbonate hemodialysis combined with hemoperfusion was performed in a standard manner. The system was flushed with saline containing
$50 \mathrm{mg}$ of Heparin. $50 \mathrm{mg}$ of Heparin was given i.v. at the beginning of the procedure. Additional Heparin was given whenever activated clotting time (ACT) was shorter than $400 \mathrm{~s}$.

About six hours after the procedure a surgeon was called to see that patient who manifested with dyspnoea and subsequently hemorrhagic shock.

The chest X-ray /Fig. 1/ showed homogenous opacity of the left hemithorax with fluid and, moreover, shifting of the mediastinum to the right. As the patient was severely shocked and hypotensive a chest tube was inserted immediately into his left pleural cavity and simultaneously massive volume replacement was begun via several venous lines. The chest tube evacuated 5 liters of hemorrhagic fluid at once and subsequent blood loss from that tube continued being about $1 / 2$ liter per 15 minutes.

Urgent left posterolateral thoracotomy / 5 th intercostal space/ was performed. The cupula region as well as the mediastinum were severely infiltrated by hematoma. Venous blood was leaking through the pleura around the first rib anteriorly. The exact suture of the injured wall of subclavian vein was not possible through that approach, so the bleeding was managed by inserting several atraumatic stitches through parietal pleura. During and after the operation the patient's circulation and coagulation parameters were improved.

Unfortunately, about 3 hours later bleeding into patient's left pleural cavity reappeared. Blood loss from the chest tube reached approximately $1 / 2$ liter per hour. Therefore we decided to operate the patient again through an access opened by transsection of the left clavicle. The skin incision was tracing the clavicle, the bone was then transsected la- 
terally from the sternocleidomastoid muscle insertion and between the medial and lateral third. Then the middle part of the clavicle was pulled downward together with the pectoral muscle flap /Fig. 2/. That access enabled us to localize and suture 3 bleeding injuries of the subclavian vein. Then under visual control we removed the double-way dialyzation cannula from the vein and the place of insertion was also sutured.

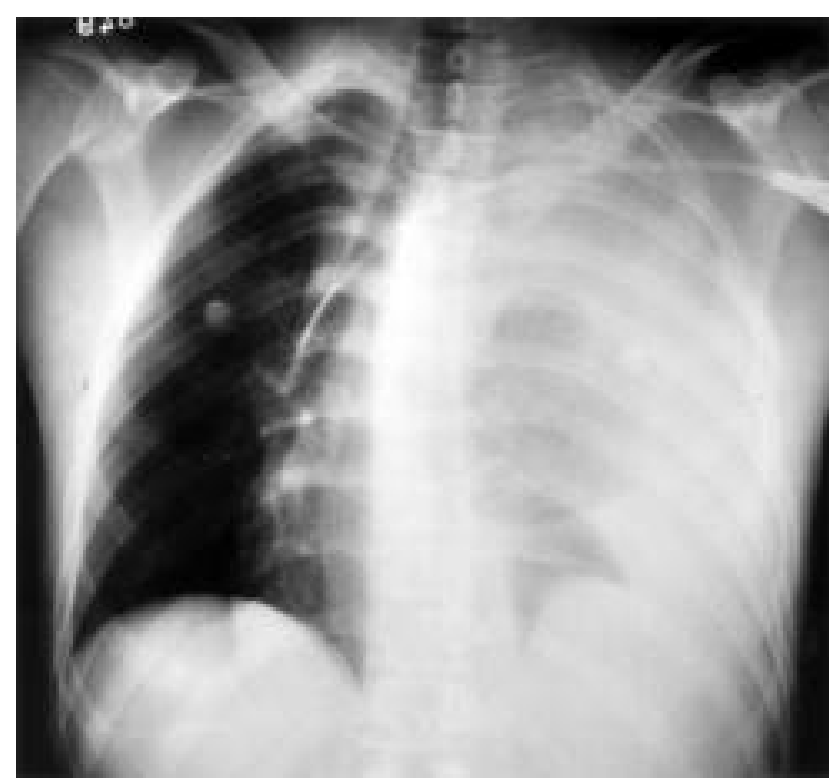

Fig. 1: Chest X-ray: Fluidothorax /hemothorax/ of the left pleural cavity with mediastinal dislodgement to the right

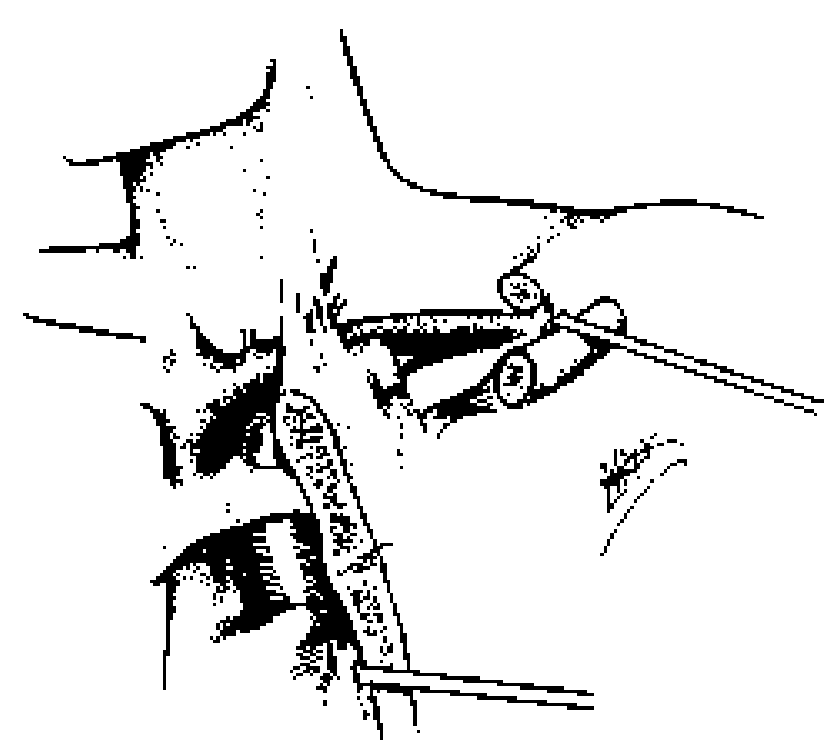

Fig. 2: Surgical access via the left clavicle and median sternotomy
In spite of that there was still blood oozing from the upper mediastinum. Due to the serious patient's clinical state we finally decided to perform median sternotomy to be able to check the brachiocephalic vein. Another vascular stitch was necessary to treat minor bleeding point on it. Osteosynthesis of the clavicle and sternum together with skin reconstruction followed.

Subsequent management in the intensive care unit was directed to restore electrolyte and fluid balance and to correct pathologic coagulation parametres. The patient was arteficially ventilated for next 8 hours and than he could be weaned from the ventilator and extubated. The further hospital course was uneventful and the patient was discharged from the hospital 10 days later.

\section{Discussion}

When hemothorax appears as a complication of subclavian vein cannulation, it can be managed conservatively by performing pleural drainage, maintaining the circulation volume by supplying blood and fluids and, last but not least, restoring coagulation parameters (4). When conservative management is not successful, surgical treatment must be used to control bleeding.

If coagulation parameters are normal, usually venous bleeding caused by venepuncture stops spontaneously. In our patient the coagulation was severely impaired by heparin administered due to dialysis. After severe blood loss and massive volume expansion by crystaloids, coloids and blood transfusions further impairment of coagulation progressed.

Lux (2) demonstrates the incidence of pneumothorax after central venous catheterization in $1,76 \%$ and arterial puncture in $4,1 \%$.

Queires (5) reports about 92 patients in whom the dialyzation cannula was inserted into subclavian vein. In this group the incidence of hemothorax was $2,2 \%$.

Because of seriously deteriorated state of our patient, who was in profound hemorrhagic shock we first chose surgical access via posterolateral thoracotomy despite being aware of the fact that such an approach might not enable exact identification of the bleeding point at the subclavian vein. Posterolateral thoracotomy, however, enabled us to stop the bleeding very quickly. There was a chance to manage the bleeding through that access assuming that the bleeding was of venous origin.

In our patient we managed to control the bleeding after the first intervention only temporarily. After the bleeding reappeared we had to perform surgical revision via supraclavicular access with partial resection of the clavicle. As further revision of the brachiocephalic vein was needed, median sternotomy was also performed. That access was more time consuming, but enabled us proper direct checking of the subclavian and brachiocephalic veins. In case of arterial bleeding this approach would enable the subclavian artery revision, as well. Only the complex surgical procedu- 
re enabled proper management of bleeding complication in presented case.

\section{References}

1. Aubaniac R. L'injection intraveineuse sous-claviculaire. Presse Med 1952;60:1656.

2. Lux EA. Der Punktionskathetrismus zentraler Venen. Erfahrungen aus einer Klinik der Grundsversorgung. Zentralbl Chir 1992;117:427-31.

3. Sznajder I, Zveibil R, Hetterman H, et al. Central Vein Catheterization. Failure and complication rates by three percutaneous approaches. Arch Intern Med 1985;146:25961.

4. Quillen K, Magarace L, Flangan J, et al. Vascular erosion caused by a double-lumen central venous catheter du- ring therapeutic plasma exchange. Transfusion 1995; 35:510-2.

5. Queieros J, Cabrita A, Maximino J, et al. Central catheters for hemodialysis: a six month experience of 103 catheters. Nephrologie 1994; $15: 113-5$.

Submitted May 1997.

Accepted May 1997.
MUDr. Jan Harrer, CSc., Department of Cardiac Surgery,

University Teaching Hospital, 50005 Hradec Králové, Czech Republic. 